\title{
Comparing multichannel cylinder and 3D-printed applicators for vaginal cuff brachytherapy with preliminary exploration of post-hysterectomy vaginal morphology
}

\author{
Junfang Yan, MD*, Xue Qin, MS*, Fuquan Zhang, MD, PhD, Xiaorong Hou, MD, Lang Yu, MS, Jie Qiu, PhD \\ Department of Radiation Oncology, Peking Union Medical College Hospital, Chinese Academy of Medical Sciences and Peking Union \\ Medical College, Beijing, China \\ *Junfang Yan and Xue Qin contributed equally to this work. \\ Department and institutional address where the research was conducted: Department of Radiation Oncology. Peking Union Medical College \\ Hospital, Chinese Academy of Medical Sciences and Peking Union Medical College, Beijing, China
}

\begin{abstract}
Purpose: To compare dosimetric parameters of multichannel cylinder (MCC) and 3-dimensional (3D)-printed plans for high-dose-rate vaginal cuff brachytherapy (VBT) and to explore vaginal morphologies after hysterectomy.

Material and methods: Forty-eight patients with gynecological cancer who required VBT after hysterectomy were investigated. To develop a 3D-printed applicator, computed tomography images were obtained with contrast-soaked vaginal gauzes placed in situ to mould vaginal anatomy. 3D digital model of vaginal gauze was then generated and subsequently converted into a prototype 3D-printed applicator, and optimized source catheters were placed in the applicator. Post-hysterectomy vaginal configurations were classified via morphometric analysis of 3D-printed applicators.
\end{abstract}

Results: In terms of clinical target volume (CTV) coverage, $\mathrm{V}_{100}$ was slightly larger in 3D-printed plans than in MCC plans $\left(90.02 \%\right.$ vs. $90.01 \%, p<0.001$ ), and $\mathrm{D}_{98}$ in 3D-printed group (429.11 cGy) was higher than that in MCC group (400.08 cGy, $p<0.001$ ). Quality indices were superior in 3D-printed plans than in MCC plans. When evaluating proximal $1 \mathrm{~cm}$ of CTV from the vaginal apex $(\mathrm{CTV}-1 \mathrm{~cm})$, difference in $\mathrm{V}_{100}$ was significant and favored 3D-printed plans $(81.85 \%$ vs. $72.47 \%, p<0.001)$. 3D-printed applicators demonstrated superiority over MCC in terms of bladder and rectum protection. However, it would cause higher undesired doses to sigmoid. Post-hysterectomy vaginal configurations were classified into five different types, including dome-column, Gothic arch-column, two dog ears-column, one dog ear-column, and 'up wide and low narrow'.

Conclusions: Compared with MCC, 3D-printed applicators delivered a higher dose and larger volume, and offered more homogeneous and conformal target coverage. Further research on vaginal morphology will be valuable to help screen out patients who need 3D-printed applicators, and to provide guidance for designing new applicators.

J Contemp Brachytherapy 2021; 13, 6: 64l-648

DOI: https://doi.org/10.5114/jcb.2021.112115

Key words: hysterectomy, vaginal cuff brachytherapy, multichannel applicator, 3D printing, vaginal morphology.

\section{Purpose}

Endometrial and cervical cancer are two of the most common gynecological malignancies that afflict women worldwide [1]. Vaginal cuff brachytherapy (VBT) has become an essential technology used for adjuvant treatment of endometrial cancer of patients who are at high-risk of recurrence post-hysterectomy $[2,3]$ and carefully selected post-hysterectomy patients with cervical cancers [4].
The selection of an appropriate VBT applicator is important to achieve optimal results.

Single-channel cylinder (SCC) is the most common applicator for VBT [5]. However, it is limited in its' ability to sculpt clinical target volume (CTV) and organs at risk (OARs) doses due to its' single-source anisotropy. On the contrary, multichannel cylinder (MCC) applicator presents with more peripheral channels to allow for dose flexibility [6]. It is reported that MCC significantly reduc-
Address for correspondence: Jie Qiu, Department of Radiation Oncology, Peking Union Medical College Hospital, Chinese Academy of Medical Sciences and Peking Union Medical College, No. 1 Shuaifuyuan Wangfujing, Dongcheng District, 100730 Beijing, China, phone: +86 10 6915 5481, fax: +86 106512 4875, ๑ e-mail: qiujie@pumch.cn
Received: 03.05.2021

Accepted: 14.10 .2021

Published: 30.12 .2021 
es doses to the rectum (by approximately 15\%) and bladder (by 1.4-14\%) compared with SCC [7-9]. At the Peking Union Medical College Hospital, we have used in-house MCC applicator for adjuvant VBT since 2003, and have observed a 5-year overall survival and local recurrence rates of $96.3 \%$ and $2.9 \%$, respectively, in patients with stage I high-intermediate-risk endometrial cancer [10]. However, post-hysterectomy vaginal morphology varies due to tumor's location, surgical approach, and surgical closure, thus cylindrical applicators cannot fit all the patients. Customized vaginal mould can adapt and show the shape of the vagina, and it can be used for irregular vaginal vault configuration [11-14].

Three-dimensional (3D) printing has enabled a physical creation of customized vaginal moulds with optimized catheters embedded in applicator. Wiebe et al. found that a 3D-printed applicator could offer favorable dose distribution at the vaginal apex for a 'dog ear' vaginal configuration, with sufficient and effective dwell positions [15]. However, previous studies investigating the use of this individual applicator in vaginal brachytherapy have focused on interstitial brachytherapy [16-22], whereas reports on vaginal intracavitary brachytherapy are scarce [15, 23]. As such, whether 3D-printed applicators are superior to MCCs in clinical practice remains unknown.

We adopted 3D-printed individualized applicators at our institution in 2015. Under a hypothesis that 3D-printed applicators would provide better CTV coverage and OARs' protection, we performed this study to compare the dosimetric parameters of 3D-printed plans for highdose-rate (HDR) VBT to those of MCC plans at a given dose. Additionally, we explored and classified post-hysterectomy vaginal morphologies via analysis of the 3D-printed applicator shapes.

\section{Material and methods}

\section{Patients' selection}

We extracted consecutive patients with gynecological cancers who required VBT after hysterectomy, and those with lesions thicker than $10 \mathrm{~mm}$ were excluded. In total, 48 patients were analyzed including 26 with endometrial cancer, 16 with cervical cancer, 3 with recurrent endometrial cancer, and 3 with recurrent cervical cancer.

\section{Applicators}

The in-house multichannel cylinder applicator (Patent No. ZL201320564893.3) comprised one central channel and six peripheral channels; its' details have been described previously [10]. The development of 3D-printed applicator is shown in Figure 1. There were three critical steps involved in generating a 3D-printed applicator: 1. Vaginal packing with gauze, in which the vagina was filled with narrow, thin gauze pre-soaked in diluted diatrizoate meglumine. Then, computed tomography (CT) images were obtained and the gauze was withdrawn, which took about 10 minutes; 2 . Extracting the gauze and placing source catheters, in which CT images were transferred to 3D Slicer software (http://www.slicer.org) to determine the volume of vagina gauze, served as a prototype of a 3D-printed applicator. The outer catheters were then placed approximately $5 \mathrm{~mm}$ away from the applicator surface, with $10 \mathrm{~mm}$ space between each other. It took about 30 minutes for image extraction and model design; 3. Production and quality assurance of 3D-printed applicator, in which individualized applicator was made from a biocompatible OBJET MED610 polymer (Stratasys Ltd., Rehovot, Israel), using Eden 260VS 3D printer for 1-2 hours. The applicator was then subjected to multiple physical evaluations to ensure its' suitability for clinical use. Additionally, we classified the 3D-printed applicators depending on their apex shapes.

\section{CT simulation and treatment plans}

All the patients received pelvic CT simulation with $3 \mathrm{~mm}$ slice thickness to confirm the position of applicators. Contouring and treatment planning were developed using Oncentra (Elekta, Stockholm, Sweden) brachytherapy treatment planning system.

\section{Dosimetric parameters}

The goal was to cover $90 \%$ of CTV using the prescribed dose of 500 cGy at $5 \mathrm{~mm}$ depth. CTV was defined as a $5 \mathrm{~mm}$ expansion beyond the vaginal applicator surface; any surrounding air gaps and lesions beyond $5 \mathrm{~mm}$ would be considered when contouring CTV, while OARs were excluded from CTV. Treated length was approximately $1 / 3$ to $1 / 2$ of the vagina, depending on pathological sub-type, International Federation of Gynecology and Obstetrics stage, and risk of recurrence.

CTV-1 cm was defined as proximal $1 \mathrm{~cm}$ of CTV from the vaginal apex, which normally represented the true vaginal cuff volume [6]. Measuring this parameter was reasonable, given that the most common location of post-operative endometrial cancer recurrence is the vaginal vault $[24,25]$. Four quality indices were evaluated, including conformity index (CI), which was defined as a ratio of volume receiving $\geq 95 \%$ of the prescription dose to total CTV $\left(\mathrm{CI}=\mathrm{V}_{95} / \mathrm{V}_{\mathrm{CTV}}\right)$; dose homogeneity index (DHI), which was defined as a ratio of volume receiving $100-150 \%$ of the prescription dose to total CTV $\left(\mathrm{DHI}=\mathrm{V}_{100-150} / \mathrm{V}_{\mathrm{CTV}}\right)$; dose non-uniformity rate (DNR), defined as a ratio of volume receiving at least $150 \%$ of the prescription dose to that receiving at least $100 \%$ of the prescription dose (DNR $\left.=\mathrm{V}_{150} / \mathrm{V}_{100}\right)$; and overdose index (OI), defined as a ratio of volume receiving at least $200 \%$ of the prescription dose to that receiving at least $100 \%$ of this dose $\left(\mathrm{OI}=\mathrm{V}_{200} / \mathrm{V}_{100}\right)$. Overall, the ideal CI value was 1 , and a higher DHI indicated a more homogeneous dose distribution in the treatment volume, while a smaller DNR showed a lower non-uniform dose distribution in the treatment volume. The ideal OI rate was 0 .

Wilcoxon signed-rank test was applied to compare differences between MCC and 3D-printed applicators. Fisher's exact probability method was used to compare the incidences of air gaps associated with different vaginal morphologies on CT images. A two-sided $p$-value $<0.05$ was considered statistically significant. 

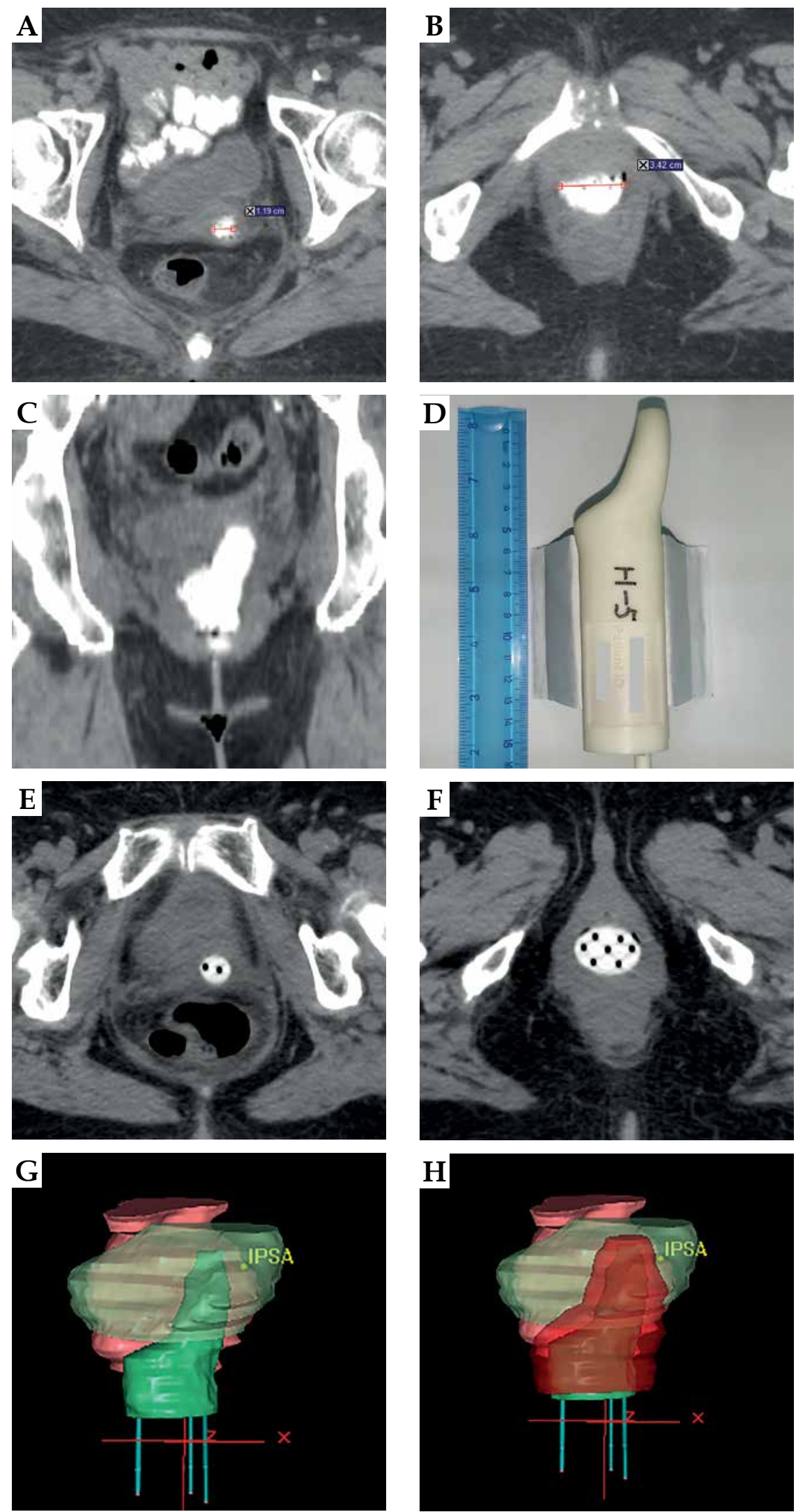

Fig. 1. A-C) Vaginal packing with contrast-soaked gauze: A) the upper region, B) the lower region, C) the coronal view. D) The corresponding 3D-printed applicator. E, F) CT images with the applicator in situ: E) the upper region, F) the lower region. $\mathbf{G}, \mathbf{H}$ ) The reconstruct volume of applicator and target in treatment planning system: $\mathbf{G}$ ) the green applicator, $\mathbf{H})$ the red clinical target volume 
Table 1. Patients' characteristics

\begin{tabular}{lc} 
Characteristics & $\begin{array}{c}\text { Number of patients } \\
(n=48)\end{array}$ \\
\hline Age (years), median (range) & $54(30-78)$ \\
\hline Diagnosis & 26 \\
\hline Endometrial cancer & 16 \\
\hline Cervical cancer & 3 \\
\hline Recurrent endometrial cancer & 3 \\
\hline Recurrent cervical cancer & 24 \\
\hline Treatment & 24 \\
\hline VBT & 24 \\
\hline EBRT + VBT & 24 \\
\hline Air gaps & $0.11(0.02-0.33)$ \\
\hline With & 21 \\
\hline Without & 12 \\
\hline Volume (cm $\left.{ }^{3}\right)$ median (range) & 6 \\
\hline Vaginal configuration & 5 \\
\hline Dome-column & 4 \\
\hline Gothic arch-column & \\
\hline Two dog ears-column & \\
\hline One dog ear-column & \\
\hline Up wide and low narrow & \\
VBT - vaginal cuff brachytherapy; EBRT-external beam radiation therapy
\end{tabular}

\section{Results}

Table 1 shows the patients' characteristics. Air gaps were present in 24 of the $48 \mathrm{MCC}$ CT simulation sets; the median air gap volume was $0.11 \mathrm{~cm}^{3}$ (range, $0.02-0.33 \mathrm{~cm}^{3}$ ). Post-hysterectomy vaginal morphologies were classified into 5 types based on 3D-printed applicator shapes (Figure 2), including dome-column, Gothic arch-column, two dog ears-column, one dog ear-column, and 'up wide and low narrow'.

Table 2 shows the comparison between dosimetric parameters of the MCC and 3D-printed plans. In terms of CTV coverage, $\mathrm{V}_{100}$ was slightly larger in the $3 \mathrm{D}$-printed plan than in the MCC plan $(90.02 \%$ vs. $90.01 \%, p<0.001)$ and $\mathrm{D}_{98}$ in the 3D-printed group (429.11 cGy) was higher than that in the MCC group (400.08 cGy, $p<0.001)$. All four quality indices (CI, DHI, DNR, and OI) were superior in the 3D-printed plans than in the MCC plans. When evaluating CTV-1 cm, the difference in $V_{100}$ was significant and favored the 3D-printed plans $(81.85 \%$ vs. $72.47 \%, p<0.001$ ). Additionally, mean $\mathrm{D}_{90}$ was significantly higher in the 3D-printed plans than in the MCC plans (470.05 vs. 422.11 cGy, $p<0.001$ ), indicating inadequate vaginal apex coverage by both applicators, especially during MCC treatment.

Table 3 illustrates the dosimetric comparison for OARs between the two plans. $\mathrm{D}_{2 \mathrm{cc}}$ and $\mathrm{D}_{0.1 \mathrm{cc}}$ of the bladder and $\mathrm{D}_{0.1 \mathrm{cc}}$ of the rectum were lower in the 3D-printed
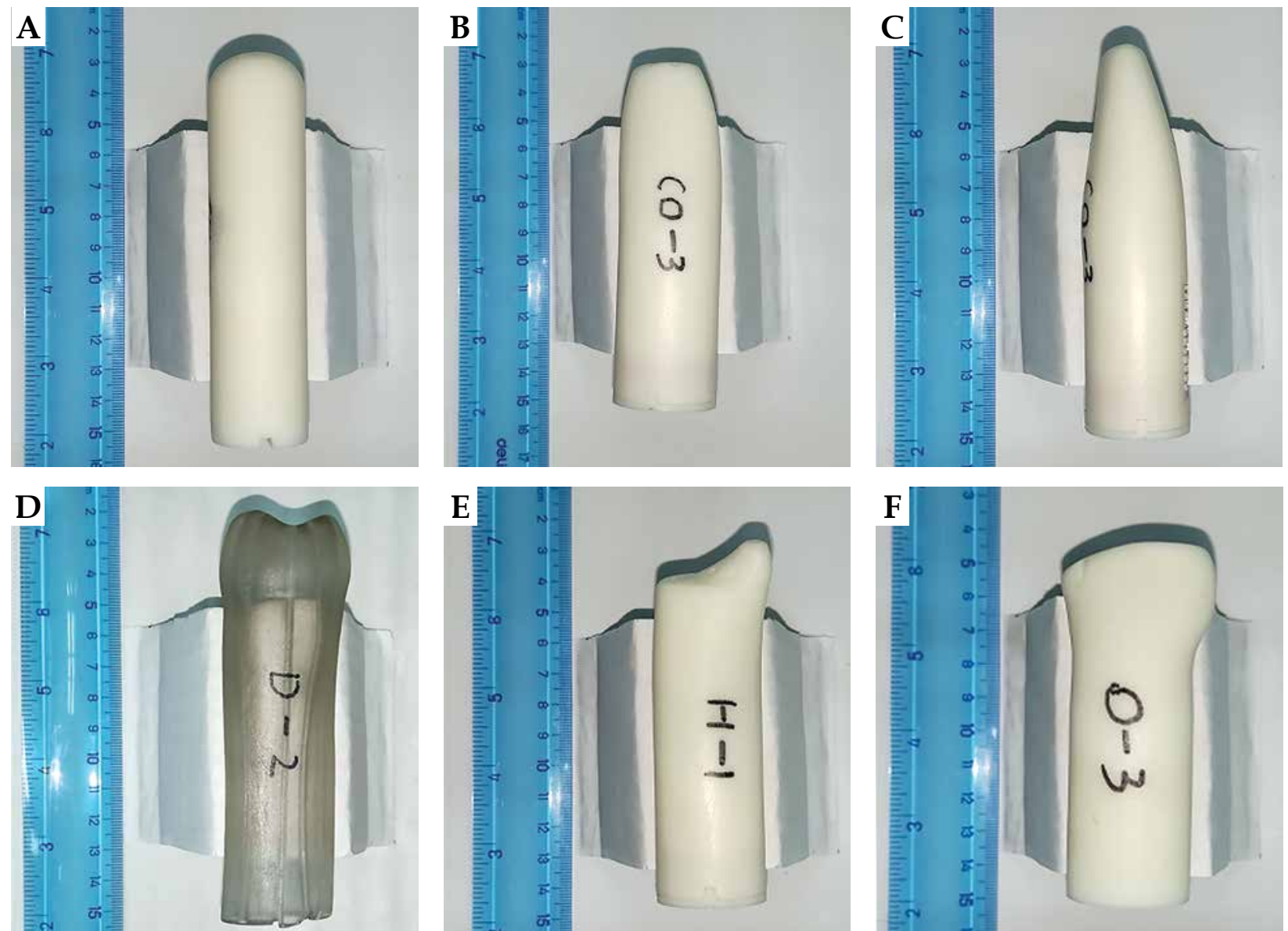

Fig. 2. Photographs of the 3D-printed applicators. A) Dome-column, B) coronal view of the Gothic arch-column, C) sagittal view of the Gothic arch-column, D) two dog ears-column, E) one dog ear-column, and F) 'up wide and low narrow' 
plan than in the MCC plan. No differences were noted between the two plans in terms of $D_{2 c c}$ of the rectum and $\mathrm{D}_{2 \mathrm{cc}}$ and $\mathrm{D}_{0.1 \mathrm{cc}}$ of the small intestine. However, $\mathrm{D}_{2 \mathrm{cc}}$ and $\mathrm{D}_{0.1 \mathrm{cc}}$ of the sigmoid in the 3D-printed group were higher than those in the MCC group (129.34 vs. 99.55 cGy, $p<0.001 ; 184.48$ vs. $154.25 \mathrm{cGy}, p=0.001$, respectively).

Table 4 shows the air gap incidences associated with the five vaginal morphologies. The two dog ears-column had the highest air gap rate at $83.3 \%$, followed by the
Gothic arch-column (75\%), one dog ear-column (40\%), dome-column $(33.3 \%)$, and 'up wide and low narrow' $(25 \%)$. The differences between these rates were not statistically significant.

\section{Discussion}

Our study was the first to perform dosimetric comparison between MCC and 3D-printed applicators for HDR

Table 2. Dosimetric comparison of clinical target volume (CTV) between multichannel cylinder (MCC) and three dimensional (3D)-printed plans. Data are presented as median (interquartile range)

\begin{tabular}{lccc} 
Dosimetric parameters & MCC applicator & 3D-printed applicator & $p$-value \\
\hline CTV & & & $<0.001$ \\
\hline $\mathrm{V}_{100}(\%)$ & $90.01(0.05)$ & $90.02(0.06)$ & $<0.001$ \\
\hline $\mathrm{D}_{98}(\mathrm{CGy})$ & $400.08(70.30)$ & $429.11(27.50)$ & $<0.001$ \\
\hline $\mathrm{Cl}$ & $0.929(0.013)$ & $0.939(0.009)$ & $<0.001$ \\
\hline DHI & $0.347(0.053)$ & $0.366(0.047)$ & $<0.001$ \\
\hline DNR & $0.615(0.059)$ & $0.594(0.053)$ & $<0.001$ \\
\hline OI & $0.393(0.087)$ & $0.343(0.104)$ & $<0.001$ \\
\hline CTV-1cm & & & $<0.001$
\end{tabular}

$\mathrm{Cl}$ - conformity index; $\mathrm{DHI}$ - dose homogeneity index; DNR - dose nonuniformity rate; Ol-overdose index; CTV-clinical target volume; CTV-1cm - proximal $1 \mathrm{~cm}$ of CTV from vaginal apex

Table 3. Dosimetric comparison of organs at risk (OARs) between multichannel cylinder (MCC) and three dimensional (3D)-printed plans. Data are presented as median (interquartile range)

\begin{tabular}{lccc} 
Dosimetric parameters & MCC applicator & 3D-printed applicator & $p$-value \\
\hline Bladder & & & 0.012 \\
\hline$D_{2 c c}(c G y)$ & $377.23(49.00)$ & $365.16(57.02)$ & $<0.001$ \\
\hline$D_{0.1 c c}(c G y)$ & $491.87(70.12)$ & $462.53(53.73)$ & 0.697 \\
\hline Rectum & & $343.85(62.52)$ & 0.001 \\
\hline$D_{2 c c}(c G y)$ & $336.64(43.73)$ & $457.05(59.16)$ & $<0.001$ \\
\hline$D_{0.1 c c}(c G y)$ & $486.35(79.06)$ & & 0.001 \\
\hline Sigmoid & & $129.34(119.42)$ & 0.129 \\
\hline$D_{2 c c}$ (cGy) & $99.55(81.66)$ & $184.48(196.44)$ & 0.538
\end{tabular}

Table 4. Incidences of air gaps for each of the five vaginal morphologies

\begin{tabular}{lccc} 
Vaginal morphology & \multicolumn{1}{c}{ Air gap } & Air gap incidence \\
\cline { 2 - 4 } & With $(n)$ & Without $(n)$ & 33.3 \\
\hline Dome-column & 7 & 14 & 75.0 \\
\hline Gothic arch-column & 9 & 3 & 83.3 \\
\hline Two dog ear-column & 5 & 1 & 40.0 \\
\hline One dog ear-column & 2 & 3 & 25.0 \\
\hline Up wide and low narrow & 1 & 3 & 50.0
\end{tabular}


VBT. Moreover, this was the first study to differentiate post-hysterectomy vaginal morphologies into 5 types via analysis of 3D-printed applicator shapes.

An ideal applicator should perfectly fit the shape of vaginal cavity and provide enough dwell positions to avoid both target underdosage and OARs overdosage. In terms of cylindrical applicators used during VBT treatment, commercial SCCs and MCCs have the same shape and size; however, the latter have more catheters that enable flexible dwell positions. MCC applicators have become significantly more popular in recent years [5, 26]; however, the vaginal anatomy is non-uniformly cylindrical. Air gaps are often observed during VBT treatment when using cylindrical applicators. One of the most central features of brachytherapy is the inverse square factor (dose rate $=\mathrm{K} /$ distance $^{2}$ ). A displacement of 1-2 mm of vaginal wall caused by an air gap can decrease the dose to vaginal mucosa by more than $10 \%$ [27]. Another study has shown that a $1 \mathrm{~mm}$ displacement of vaginal mucosa would result in a mean dose reduction of $7.3 \%$ and $9.2 \%$ at a $5 \mathrm{~mm}$ depth and at vaginal surface, respectively [28]. Choo et al. has found that more than $90 \%$ of lymphatics lie within $2-3 \mathrm{~mm}$ of stretched vaginal mucosal surface [29]. Therefore, HDRVBT was prescribed at a $5 \mathrm{~mm}$ depth from the applicator surface to allow for a $2 \mathrm{~mm}$ tolerance [30].

If air gaps larger than $2 \mathrm{~mm}$ cannot be reduced, customized applicators may provide a solution. Magné et al. reported on a vaginal mould applicator, which can allow for tailored treatment in terms of morphologic optimization [12]. There were some procedures for this vaginal mould: 1 . A vaginal impression was made to outline patient's anatomy and tumor topography; 2 . A rough hollow acrylic applicator was made; and 3. The position of catheters was confirmed to provide satisfactory dosimetric coverage. The effect of vaginal impression made from an alginate material is similar to that of our vagina packing with thin strips of gauze. Handling of chemicals in the vaginal mould-making process was not easy for the staff, but 3D-printed applicator did not have this problem. Similar to MCC, molded applicator flattened the irregular vaginal apex by expanding the vaginal wall [13]. It may be uncomfortable and difficult for vaginal mould to flatten the long "dog-ear" configuration (Figure 1D), but easy for the 3D-printed applicator to adapt to this configuration without discomfort. In addition, for some 'up wide and low narrow' configuration patients, it may be difficult to extract the vaginal impression from the vagina once it has hardened, but easy to remove the thin gauze strips one by one. Furthermore, the 3D-printed applicator can be designed with two parts to be inserted into the narrow vaginal introitus with minimal patient's discomfort. Wiebe et al. also reported similar two-part design [15]. Additionally, the arrangement of catheters should be of special interest. Our 3D-printed applicator had a circle of peripheral catheters and several central catheters; therefore, there were more catheters than 3 in Magné et al.'s, and more flexible arrangement than that in Wiebe et al.'s linear arrangement. Furthermore, for patients with obvious residual lesions before VBT, the catheters can be preliminarily designed by radiation oncologists and physicists based on CT images with vaginal gauze. Then, target coverage and dose distribution can be roughly evaluated before the 3D-printed applicator is made.

Our results confirmed that 3D-printed applicators could cover a larger CTV than MCC applicators, especially CTV $-1 \mathrm{~cm}$. Since the optimization condition was limited to $\mathrm{D}_{90}=500 \mathrm{cGy}$, the improvement in CTV $\mathrm{V}_{100}$ was only $0.01 \%$. However, the 3D-printed applicators offered higher $D_{98}$ and more homogeneous dose distributions within CTV. Additionally, our study showed significant improvements in CTV-1 cm V $\mathrm{V}_{100}$ (by $13 \%$ ) and $\mathrm{D}_{90}$ (by $11 \%$ ) when compared with MCC. When comparing the 3D-printed applicators to standard SCC counterparts in patients with 'dog ear' vaginal configuration, Wiebe et al. found that CTV $\mathrm{V}_{100}$ improved by $13.2 \%$, whereas the dose of 'two ears' area increased by $113 \%$, with more uniformity $\left(\mathrm{V}_{200}\right.$ reduction of $\left.6.8 \%\right)$. Simultaneously, total doses to OARs when using 3D-printed applicators were lower than those observed when using SCCs [15]. In our study, the 3D-printed applicators showed superiority over MCCs in terms of the bladder and rectum protection. However, we found it would cause higher undesired doses to the sigmoid. It is possible that better coverage of CTV-1 cm was achieved at the expense of a higher dose to the sigmoid.

Although vaginal anatomy was usually considered as a cylinder during VBT treatment, it has been found to resemble a funnel [31]. From the vaginal introitus to the cervix, the distended vagina was consistently found to have three zones: a superficial sphincteric narrow zone, a central wedge-shaped transition zone, and an expanded forniceal zone. The narrow introitus limited the size of applicator; hence, air gaps were likely to occur at the expanded fornix when using cylindrical applicators with a suitable diameter for the vagina introitus. Even if no air gap was observed on CT image, the vaginal folds at the fornix were often piled up, which goes against the principle of stretching the vaginal mucosa as much as possible during VBT treatment. The 3D-printed applicator can conformally fit into the three consistent zones and extend the vaginal folds without discomfort. Various surgical approaches may deviate from the vaginal axis and affect the angle between the upper and lower vaginal segments [32], thereby affecting the shape of the vaginal cuff $[33,34]$. Park et al. found that vaginas demonstrated an elliptical rather than circular cross-sectional anatomy [9]. As the configuration of vagina is complex, we failed to find quantitative indicators for a classification of 3D-printed applicators, and roughly divided them into five types to show vaginas' morphologies.

The dome-column $(43.8 \%)$ was the most commonly observed vaginal morphology post-hysterectomy in this study, and the best-suited for cylinder applicators. The Gothic arch-column, two dog ears-column, and one dog ear-column configurations of the vaginas, which presented with high air gap rates when using MCC, may preferably use a 3D-printed applicator. We also found that it was clinically feasible to judge the vaginal morphology through gynecological examinations before CT simulation. For example, it was easy to differentiate two dog ears-column configurations in gynecological examina- 
tions, indicating that those patients may need a 3D-printed applicator. However, no significant differences were found in air gap incidences among the five vaginal morphologies, partially owing to the small sample size.

The thickness of lesions treated in this study was limited to $10 \mathrm{~mm}$. For patients with tumor sizes $>10 \mathrm{~mm}$ [18], paravaginal extension or pararectal space involvement [16], and insufficient target coverage [20], combined intracavitary/interstitial techniques should be used. Novel 3D-printed intracavitary applicators based on intensity-modulated techniques has been shown to be clinically feasible for treating vaginal tumors thicker than $10 \mathrm{~mm}$ [35]. These applicators can potentially improve therapeutic ratio by facilitating OARs' dose reduction, CTV dose escalation, or both [36].

There were limitations in this study. Our in-house MCCs only had a single-fixed diameter of $26 \mathrm{~mm}$; however, this was suitable for most patients treated at our center. Furthermore, the incidence of air gaps on MCC CT images was 50\%, which was lower than that reported by Cameron et al. [37] and Richardson et al. [38], but similar to Hassouna et al.'s reported rate of 58\% [27], which indicated good contact between in-house MCC and vagina. Secondly, the relatively small sample size confounded the identification of vaginal morphology most likely to generate air gaps. Thirdly, it was a retrospective study.

\section{Conclusions}

Compared to MCC, the 3D-printed applicators delivered a higher dose and larger volume, and offered more homogeneous and conformal target coverage. Further research on vaginal morphology would be valuable to help screen out patients who require 3D-printed applicators, and also to provide guidance for a design of new applicators.

\section{Funding}

This work was partially supported by grants from the National Key Research and Development Plan, the Ministry of Science and Technology of the People's Republic of China (Grant No. 2016YFC0105206), the Non-Profit Central Research Institute Fund of Chinese Academy of Medical Sciences (Grant No. 2019XK320046), and the Young Scientific Research Program (Grant No. pumch201910569) of Peking Union Medical College.

\section{Acknowledgements}

We would like to thank Zhequn Liu of the Intelligent Manufacturing Institute, Heilongjiang Academy of Sciences, for technical support.

\section{Disclosure}

The authors report no conflict of interest.

\section{References}

1. Sung H, Ferlay J, Siegel RL et al. Global cancer statistics 2020: GLOBOCAN estimates of incidence and mortality world- wide for 36 cancers in 185 countries. CA Cancer J Clin 2021; 71: 209-249.

2. Colombo N, Creutzberg C, Amant $\mathrm{F}$ et al. ESMO-ESGOESTRO Consensus Conference on Endometrial Cancer: diagnosis, treatment and follow-up. Ann Oncol 2016; 27: 16-41.

3. Zakem SJ, Robin TP, Smith DE et al. Evolving trends in the management of high-intermediate risk endometrial cancer in the United States. Gynecol Oncol 2019; 152: 522-527.

4. Koh WJ, Abu-Rustum NR, Bean S et al. Cervical Cancer, Version 3.2019, NCCN Clinical Practice Guidelines in oncology. J Natl Compr Canc Netw 2019; 17: 64-84.

5. Harkenrider MM, Grover S, Erickson BA et al. Vaginal brachytherapy for postoperative endometrial cancer: 2014 Survey of the American Brachytherapy Society. Brachytherapy 2016 ; 15: 23-29.

6. Hou X, Liu A, Zhang F et al. Dosimetric advantages of using multichannel balloons compared to single-channel cylinders for high-dose-rate vaginal cuff brachytherapy. Brachytherapy 2016; 15: 471-476.

7. Demanes DJ, Rege S, Rodriquez RR et al. The use and advantages of a multichannel vaginal cylinder in high-dose-rate brachytherapy. Int J Radiat Oncol Biol Phys 1999; 44: 211-219.

8. Bahadur YA, Constantinescu C, Hassouna AH et al. Single versus multichannel applicator in high-dose-rate vaginal brachytherapy optimized by inverse treatment planning. J Contemp Brachytherapy 2015; 6: 362-370.

9. Park SJ, Chung M, Demanes DJ et al. Dosimetric comparison of 3-dimensional planning techniques using an intravaginal multichannel balloon applicator for high-dose-rate gynecologic brachytherapy. Int J Radiat Oncol Biol Phys 2013; 87: 840-846.

10. Hou XR, Yu L, Hu K et al. Outcomes of intermediate-risk to high-risk stage I endometrial cancer: 10-year clinical experiences of using in-house multi-channel applicators in a single center. Chin Med J (Engl) 2019; 132: 1935-1941.

11. Nilsson S, Moutrie Z, Cheuk R et al. A unique approach to high-dose-rate vaginal mold brachytherapy of gynecologic malignancies. Brachytherapy 2015; 14: 267-272.

12. Magné $\mathrm{N}$, Chargari $\mathrm{C}$, SanFilippo $\mathrm{N}$ et al. Technical aspects and perspectives of the vaginal mold applicator for brachytherapy of gynecologic malignancies. Brachytherapy 2010; 9: 274-277.

13. El Khoury C, Dumas I, Tailleur A et al. Adjuvant brachytherapy for endometrial cancer: advantages of the vaginal mold technique. Brachytherapy 2015; 14: 51-55.

14. Towithelertkul C, Chugh A, Hattori M et al. A custom-made brachytherapy applicator for recurrent endometrial and vaginal cancer: A dental technique for prosthesis fabrication. J Prosthet Dent 2021; 126: 711-714.

15. Wiebe E, Easton H, Thomas G et al. Customized vaginal vault brachytherapy with computed tomography imaging-derived applicator prototyping. Brachytherapy 2015; 14: 380-384.

16. Logar HBZ, Hudej R, Šegedin B. Development and assessment of 3D-printed individual applicators in gynecological MRI-guided brachytherapy. J Contemp Brachytherapy 2019; 11: 128-136.

17. Sekii S, Tsujino $\mathrm{K}$, Kosaka $\mathrm{K}$ et al. Inversely designed, 3D-printed personalized template-guided interstitial brachytherapy for vaginal tumors. J Contemp Brachytherapy 2018; 10: 470-477.

18. Tien CJ, Chen ZJ. A prototype open-ended multichannel intracavitary-interstitial hybrid applicator for gynecological high-dose-rate brachytherapy. Radiol Phys Technol 2020; 13: 187-194.

19. Kunogi H, Yamaguchi N, Sasai K. Evaluation of a new bivalve vaginal speculum applicator design for gynecologic interstitial brachytherapy. J Contemp Brachytherapy 2020; 12: 27-34. 
20. Logar H, Hudej R, Kobav M. 86 3D-printed multi-channel vaginal applicator for brachytherapy in gynecological cancer. Int J Gynecol Cancer 2020; 30: A102-A103.

21. Lindegaard JC, Madsen ML, Traberg A et al. Individualised 3D printed vaginal template for MRI guided brachytherapy in locally advanced cervical cancer. Radiother Oncol 2016; 118: 173-175.

22. Laan RC, Nout RA, Dankelman J et al. MRI-driven design of customised 3D printed gynaecological brachytherapy applicators with curved needle channels. 3D Print Med 2019; 5: 8.

23. Sethi R, Cunha A, Mellis K et al. Clinical applications of custom-made vaginal cylinders constructed using three-dimensional printing technology. J Contemp Brachytherapy 2016; 8: 210-216.

24. Creutzberg CL, van Putten WL, Koper PC et al. Surgery and postoperative radiotherapy versus surgery alone for patients with stage- 1 endometrial carcinoma: multicentre randomised trial. PORTEC Study Group. Post Operative Radiation Therapy in Endometrial Carcinoma. Lancet 2000; 355: 1404-1411.

25. Keys HM, Roberts JA, Brunetto VL et al. A phase III trial of surgery with or without adjunctive external pelvic radiation therapy in intermediate risk endometrial adenocarcinoma: a Gynecologic Oncology Group study. Gynecol Oncol 2004; 92: 744-751.

26. Small W, Jr., Erickson B, Kwakwa F. American Brachytherapy Society survey regarding practice patterns of postoperative irradiation for endometrial cancer: current status of vaginal brachytherapy. Int J Radiat Oncol Biol Phys 2005; 63: 1502-1507.

27. Hassouna A, Bahadur YA, Constantinescu C. Assessment of air pockets in high-dose-rate vaginal cuff brachytherapy using cylindrical applicators. J Contemp Brachytherapy 2014; 6: 271-275.

28. Abdollahi S, Rafat Motavali L, Miri Hakimabad SH, et al. (Eds.). Statistical and dosimetric analysis of air gaps in vaginal cuff brachytherapy 2017.

29. Choo JJ, Scudiere J, Bitterman P et al. Vaginal lymphatic channel location and its implication for intracavitary brachytherapy radiation treatment. Brachytherapy 2005; 4: 236-240.

30. Kirisits C, Rivard MJ, Baltas D et al. Review of clinical brachytherapy uncertainties: analysis guidelines of GEC-ESTRO and the AAPM. Radiother Oncol 2014; 110: 199-212.

31. Appelbaum AH, Zuber JK, Levi-D'Ancona R et al. Vaginal anatomy on MRI: new information obtained using distention. South Med J 2018; 111: 691-697.

32. Senturk MB, Kilicci C, Aydin S et al. Vaginal axis on MRI after unilateral and bilateral sacral hysteropexy: a controlled study. J Obstet Gynaecol 2018; 38: 115-120.

33. Nezhat C, Kennedy Burns M, Wood M et al. Vaginal cuff dehiscence and evisceration: a review. Obstet Gynecol 2018; 132 972-985.

34. Muallem MZ, Jöns T, Seidel N et al. A concise paradigm on radical hysterectomy: the comprehensive anatomy of parametrium, paracolpium and the pelvic autonomic nerve system and its surgical implication. Cancers (Basel) 2020; 12: 1839.

35. Biltekin F, Akyol HF, Gültekin M et al. 3D printer-based novel intensity-modulated vaginal brachytherapy applicator: feasibility study. J Contemp Brachytherapy 2020; 12: 17-26.

36. Callaghan CM, Adams Q, Flynn RT et al. Systematic review of intensity-modulated brachytherapy (IMBT): static and dynamic techniques. Int J Radiat Oncol Biol Phys 2019; 105: 206-221.

37. Cameron AL, Cornes P, Al-Booz H. Brachytherapy in endometrial cancer: quantification of air gaps around a vaginal cylinder. Brachytherapy 2008; 7: 355-358.
38. Richardson S, Palaniswaamy G, Grigsby PW. Dosimetric effects of air pockets around high-dose rate brachytherapy vaginal cylinders. Int J Radiat Oncol Biol Phys 2010; 78: 276-279. 